\title{
Interpersonal frontopolar neural synchronization in group communication: an exploration toward fNIRS hyperscanning of natural interactions
}

Takayuki Nozawa ${ }^{a,}$, Yukako Sasaki ${ }^{\text {b }}$, Kohei Sakaki ${ }^{b}$, Ryoichi Yokoyama ${ }^{\text {b,c }}$, Ryuta Kawashima ${ }^{a, b}$

${ }^{a}$ Department of Ubiquitous Sensing, Institute of Development, Aging and Cancer, Tohoku University, Sendai, 980-8575, Japan

${ }^{\mathrm{b}}$ Department of Functional Brain Imaging, Institute of Development, Aging and Cancer, Tohoku University, Sendai, 980-8575, Japan

${ }^{\mathrm{c}}$ School of Medicine, Kobe University, Kobe, 650-0017, Japan

*Corresponding author.

Department of Ubiquitous Sensing, IDAC, Tohoku University, 4-1 Seiryo-machi, Aoba-ku, Sendai 980-8575, Japan. Tel/Fax: +81-22-717-7988

E-mail: takayuki.nozawa.b5@tohoku.ac.jp 


\section{Abstract}

Research of interpersonal neural synchronization (INS) using functional near-infrared spectroscopy (fNIRS) hyperscanning is an expanding nascent field. This field still requires the accumulation of findings and establishment of analytic standards. In this study, we therefore intend to extend fNIRS-based INS research in three directions: (1) verifying the enhancement of frontopolar INS by natural and unstructured verbal communication involving more than two individuals; (2) examining timescale dependence of the INS modulation; and (3) evaluating the effects of artifact reduction methods in capturing INS. We conducted an fNIRS hyperscanning study while 12 groups of four subjects were engaged in cooperative verbal communication. Corresponding to the three objectives, our analyses of the data (1) confirmed communication-enhanced frontopolar INS, as expected from the region's roles in social communication; (2) revealed the timescale dependency in the INS modulation, suggesting the merit of evaluating INS in fine timescale bins; and (3) determined that removal of the skin blood flow component engenders substantial improvement in sensitivity to communication-enhanced INS and segregation from artifactual synchronization, and that caution for artifact reduction preprocessing is needed to avoid excessive removal of the neural fluctuation component. Accordingly, this study provides a prospective technical basis for future hyperscanning studies during daily communicative activities. 
Keywords: artifact reduction; fNIRS; hyperscanning; interpersonal neural synchronization; skin blood flow; social cognition 


\section{Introduction}

The functional study of the human brain has recently addressed both conventional function mapping and functional connectivity of regions within an individual brain, and the dynamic relationship of neural activities between multiple brains of interacting individuals. The rapidly growing interest in interpersonal neural synchronization (INS) by measuring socially communicating multiple brains, termed "hyperscanning", has been reflected in the number of review articles in the field (Babiloni and Astolfi, 2014; Hari and Kujala, 2009; Hasson et al., 2012; Koike et al., 2015; Konvalinka and Roepstorff, 2012; Schilbach et al., 2013). Functional magnetic resonance imaging (fMRI) studies (Bilek et al., 2015; Koike et al., 2016; Schippers et al., 2010; Stephens et al., 2010; Tanabe et al., 2012) and electroencephalography (EEG) studies (Astolfi et al., 2010; De Vico Fallani et al., 2010; Dumas et al., 2010; Kawasaki et al., 2013; Konvalinka et al., 2014; Lindenberger et al., 2009; Sanger et al., 2012) have shown that verbal and nonverbal communication induces interpersonal relationships among neural activities. Remarkably, it has been shown that the level of the interpersonal neural synchronization (INS) correlates with the level of understanding between partners (Stephens et al., 2010), a psychometric measure of social expertise (Bilek et al., 2015), and enhancement of a behavioral index of shared attention (Koike et al., 2016), which underscores the importance of investigating INS for facilitating communication and interactions. 
Functional near-infrared spectroscopy (fNIRS) is a promising method for investigating the relationship between interpersonal interactions in natural settings and their neural activities on account of its cost-effectiveness, low constraints on measurements, and relatively high tolerance of head/body motion (Egetemeir et al., 2011; Scholkmann et al., 2013). Indeed, an increasing number of fNIRS hyperscanning studies have been recently published. These studies investigate INS during interactions, both verbal (Holper et al., 2013; Jiang et al., 2015; Jiang et al., 2012), semi-verbal (cooperative singing/humming (Osaka et al., 2015)), and nonverbal types (Cui et al., 2012; Dommer et al., 2012; Funane et al., 2011; Holper et al., 2012). Consistent with other studies using fMRI or EEG, these studies have revealed a general increase of INS during communications or cooperative and engaging interactions. This suggests the possible contribution of INS to successful communication.

In exploiting the strength of fNIRS and applying the hyperscanning method to various forms of unconstrained, real-life communications/interactions, however, both further evidence accumulation and methodological refinements are required. The present study therefore intends to extend fNIRS-based research of neural synchronization during communication in three directions: (1) verifying the enhancement of frontopolar INS by natural and unstructured verbal communication involving more than two individuals; (2) examining timescale dependence of the INS 
modulation induced by communication; and (3) evaluating the effects of artifact reduction methods in capturing INS using fNIRS.

Regarding the first objective, although some recent studies have begun extending it into natural triadic communication (Jiang et al., 2015) and interactions involving additional individuals (Duan et al., 2015), most previous studies have focused on dyadic and controlled forms of communication. Unstructured interactions involving more than two individuals entail aspects that do not exist in such controlled dyadic communication. They are highly dynamic processes (Konvalinka and Roepstorff, 2012) where roles and directions become indistinct, and allows moments when some participants are not taking overt communicative actions. In such situations, relative to the processes directly causing overt communicative actions, the roles of implicit social cognitive processes can increase their importance. In this regard, the medial rostral prefrontal cortex to the frontopolar cortex, or Brodmann Area (BA) 10, has been implicated in processes that are deemed essential for successful communication, such as mentalizing; understanding the beliefs, desires, and goals of others; and multi-task coordination (Amodio and Frith, 2006; Gilbert et al., 2006; Stephens et al., 2010). It is thus expected to show enhanced INS during cooperative communication. However, the results on the enhancing effect of cooperative communication on INS in the frontopolar region have been inconclusive among the fNIRS hyperscanning studies that included that region in the measurement 
(Cheng et al., 2015; Cui et al., 2012; Dommer et al., 2012; Funane et al., 2011; Jiang et al., 2012). Therefore, we intend to verify the hypothesis that interpersonal synchronization in the frontopolar neural activities increases during a cooperative and unstructured verbal communication involving more than two individuals.

For the second objective, previous INS studies using fNIRS captured INS by averaging synchronization measures over a relatively wide-ranging timescale. In addition, the timescale range for averaging INS considerably differed among the studies. In particular, among the studies that used the wavelet transform coherence method to evaluate INS, as in the present study, Cui et al., and later Cheng et al., averaged the coherence estimates over a task-related band period of $3.212 .8 \mathrm{~s}$ or its equivalent of $0.30 .08 \mathrm{~Hz}$ (Cheng et al., 2015; Cui et al., 2012). Osaka et al. also focused on the same band based on the observation that their singing/humming task was performed on that timescale range (Osaka et al., 2015). Furthermore, Holper et al. averaged over a task-related band of $24 \mathrm{~s}(0.250 .5 \mathrm{~Hz})$ and additionally a heart rate band of $0.41 \mathrm{~s}$ (1 $2.5 \mathrm{~Hz}$ ) for comparison (Holper et al., 2012). Dommer et al. likewise averaged INS on two bands of low-frequency oscillations at $0.060 .2 \mathrm{~Hz}$ and a heart rate frequency of $0.74 \mathrm{~Hz}$ (Dommer et al., 2012). However, in more natural and unstructured interactions, especially involving more than two individuals without fixed roles such as sender and receiver, relevant behavioral and cognitive processes may distribute over 
multiple frequencies and defining a task-related band can become difficult or less appropriate. Meanwhile, Jiang et al. used a slow-frequency band for averaging either $0.010 .1 \mathrm{~Hz}$ (Jiang et al., 2012) or $0.020 .2 \mathrm{~Hz}$ (Jiang et al., 2015) in investigating natural verbal communication. On the other hand, heterogeneous contributions of different sub-scales of low-frequency oscillations ( $\mathrm{LFO}<0.1 \mathrm{~Hz}$ ) on functional network formation and psychological functions have been indicated by recent fMRI (Baria et al., 2011; Chang and Glover, 2010; Xue et al., 2014; Zuo et al., 2010) and fNIRS studies (Nakao et al., 2013; Sasai et al., 2011). In addition, recent resting-state fMRI studies indicated the functional contribution of higher-frequency $(>0.1 \mathrm{~Hz})$ fluctuations in blood oxygen level dependent (BOLD) signals (Chen and Glover, 2015; Gohel and Biswal, 2015). Therefore, to investigate the possibility of timescale-dependent modulation of INS by communication, and to avoid the risk of smearing out significant effects by averaging over wide timescales, we examined the effect of communication on INS in finer timescale bins over a wide range. This approach was also expected to be useful in clarifying how the artifact reduction methods, which are introduced below, influence the estimation of INS differently over timescales.

In terms of the third objective, fNIRS hyperscanning of natural, unconstrained communication is expected to be more prone to artifacts than in well-controlled experimental settings, supporting the importance of artifact reduction methods. Previous 
studies investigated effectiveness of artifact reduction methods for better detection of task-evoked responses (Brigadoi et al., 2014; Cooper et al., 2012; Robertson et al., 2010). However, the effectiveness of those methods for examination of INS has not been sufficiently explored to date. In this study, we focused on the effect of two methods: dual source-detector (S-D) regression to compensate the skin blood-flow component, and discrete wavelet-based motion artifact reduction (WBMR). Both methods have been reported as highly effective for evoked-response studies.

An important point to note is that the fNIRS measurement is highly sensitive to blood-flow changes in the scalp that are unrelated to brain activation and more susceptible to systemic noises and motion-induced artifacts (Haeussinger et al., 2014; Kirilina et al., 2012; Takahashi et al., 2011; Toronov et al., 2001a). Consideration of this issue is particularly important for studying neural synchrony in daily-life communications, which are more dynamic and involve more bodily and autonomic activities compared to well-controlled laboratory experiments. An approach to addressing this issue is to add a measurement of the superficial skin blood-flow signal and to exclude the corresponding component contained in the fNIRS measurement using the dual/multiple S-D regression or other similar methods. Previous studies have shown that this type of combining S-D pairs of different distances is very helpful in removing global artifacts induced by motion, cardiac and respiratory activities, or 
autonomic nervous system changes, and it thus improves the signal-to-noise ratio (Luu and Chau, 2009; Robertson et al., 2010; Saager and Berger, 2005; Saager et al., 2011; Zhang et al., 2009). Nevertheless, its effectiveness has not been proved for evaluation of INS, although a review suggested that its use is desirable for future studies (Scholkmann et al., 2013). We expected that the dual S-D regression would improve the sensitivity for enhanced INS by facilitating the dissociation of frontopolar neural signals from artifact components.

WBMR method (Molavi and Dumont, 2012) uses discrete wavelet transform to detect and remove outliers, often expressed as spike-like artifacts on a time course. It was shown to be one of the most effective methods for noise reduction and improvement of sensitivity for task-evoked activation (Brigadoi et al., 2014; Cooper et al., 2012); however, its effectiveness has not been systematically investigated for evaluation of INS. Since natural verbal communications can be more prone to head or facial motions, we expected that the WBMR method would contribute to reducing spike artifacts induced by such motions, leading to better detection of INS

\section{Materials and Methods}

\subsection{Participants}

Twelve groups of four members, totaling 48 healthy, right-handed Japanese 
university students (20 females and 28 males; age range of 19-24 years; mean 21.9), participated in the experiment. Handedness was evaluated using the Edinburgh Handedness Inventory (Oldfield, 1971). All subjects had normal or corrected-to-normal vision and reported no history of neurological or psychiatric conditions. Except two groups, all participants in each group were acquainted with each other. The constitution of each group, along with the information of their acquaintance with each other, is provided in the supplementary Table S1. This study was approved by the Ethics Committee of Tohoku University Graduate School of Medicine and was conducted according to the Declaration of Helsinki. Written informed consent was obtained from all participants.

\subsection{Tasks and procedures}

We adopted a modified Japanese cooperative word-chain game as the communication task. In this game, a player must state a word that begins with the last two syllables of the word given by the previous player (e.g., "SHI-RI-TO-RI" can be followed by "TO-RI-KA-GO"). Unlike the competitive manner in which the word-chain game is commonly played, participants were instructed to cooperate with other members and produce a word chain that is as long as possible. After one member provided a word, any member could take the next place. The aim of adopting this task was to reproduce social aspects of creative and productive group meetings in the real 
world, without requiring specific background knowledge or expertise. To successfully conduct this task, participants were required to continue guessing about the other members' thoughts and knowledge.

In each group, participants were seated around a table and faced each other (Figure 1(a)). They were instructed to take a relaxed posture in the seat and to maintain it as long as possible during the experiment. Each group session lasted $15 \mathrm{~min}$ and was divided into three blocks of 5 min each. During the first and third blocks, referred to as the "Communicating" blocks, participants conducted the cooperative word-chain game described above. During the second block referred to as the "Independent" block, which served as the baseline for the analysis of INS, participants were instructed to continue silently considering the continuation of the word chain. In doing so, they were to gaze away from the other members and avoid verbal communication, eye contact, or any other bodily interaction. Each group conducted two group sessions; each session was followed by a rest period of more than $10 \mathrm{~min}$ between them. Before the first session, to become familiar with the given task, the subjects participated in a practice session of 5 min with no recording. For each group, words that were used once could not be repeated in the same session, nor in the following sessions. Proper nouns were allowed only if all members in the group recognized them.

In addition to the NIRS brain measurement, which is described in the next section, 
the task sessions were video-recorded by four video cameras positioned behind each participant. From the recording, the time of utterance events during Communicating blocks were extracted in the time resolution of $1 \mathrm{~s}$ by the experimenters. The utterance frequency (average number of utterances per unit of time) and the distribution of inter-utterance intervals were calculated for each person and each group. In addition, the timescale characteristic of the utterance-silence time series was analyzed using a wavelet-based spectral analysis, which is explained below. The video recording failed in the first Communicating block of one session in a group; therefore, the corresponding data were excluded from the behavioral analysis of the utterance characteristics.

(a)

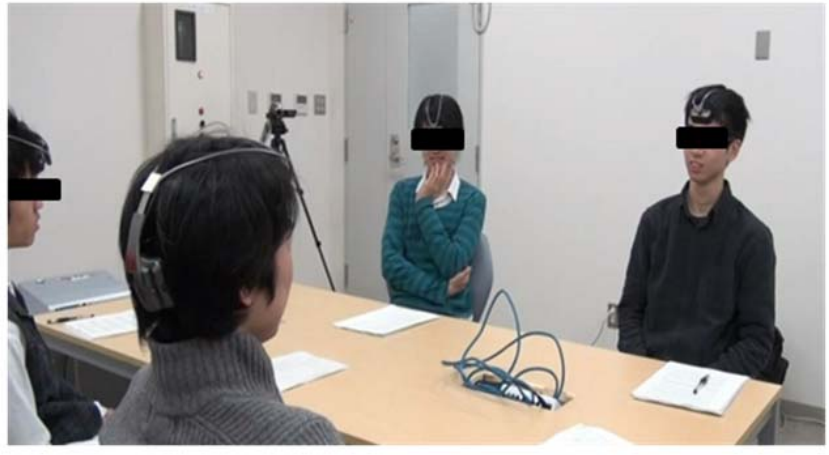

(b)
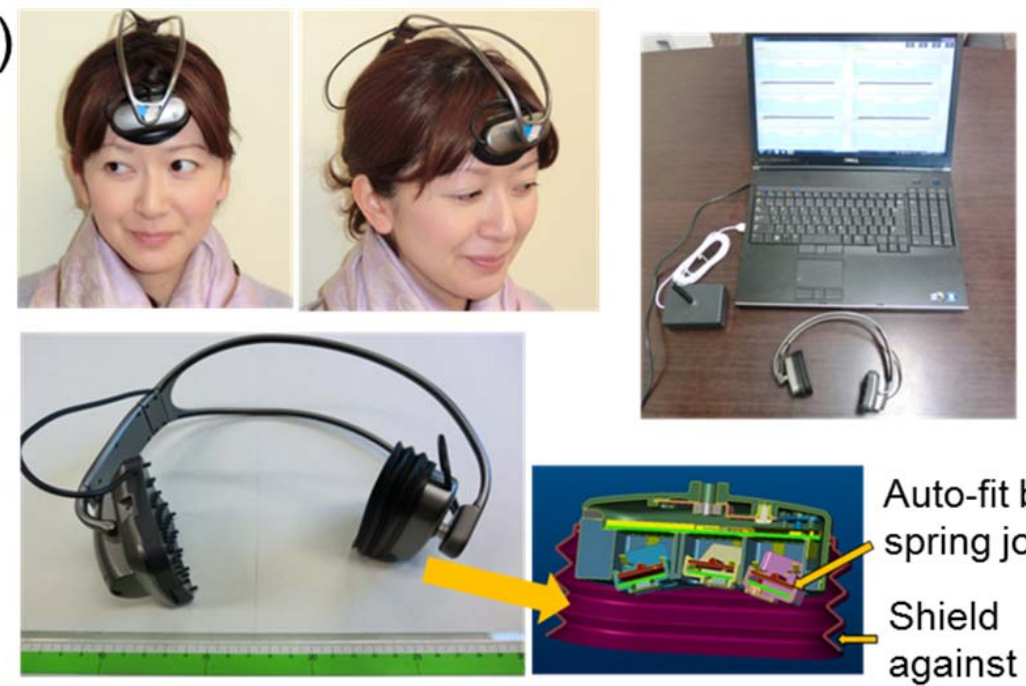

Auto-fit by spring joint

Shield against light 
Figure 1. (a) Snapshot of the communication experiment; (b) wireless fNIRS device used in this study.

\subsection{NIRS measurement}

Prefrontal neural activities of participants were recorded using a wireless continuous-wave fNIRS device (Figure 1(b)), which was developed through a collaboration of Tohoku University and Hitachi Ltd. under the support of Japan Society and Technology Agency's “Development of Advanced Measurement and Analysis Systems" program (http://www.hitachi.com/New/cnews/110914.pdf). The device consists of a front part containing optical sensor components, an adjustable and elastic arm, and a back part enclosing a wireless communication module and a battery. The outer size of the device is $215 \times 80 \times 140 \mathrm{~mm}$ and weighs $100 \mathrm{~g}$, including the battery. The optical system is comprised of a light source (a light emitting diode with the effective wavelength of $810 \mathrm{~nm}$ and the spectral half width of $35 \mathrm{~nm}$ ) and two light detectors (PN photodiodes with amplifiers), which are placed at the distance of $1.0 \mathrm{~cm}$ and $3.0 \mathrm{~cm}$ from the source, respectively. These two S-D pairs enable the dual S-D regression artifact reduction method, as explained below. The power is provided by a 3.0-V battery, which enables continuous data acquisition for $300 \mathrm{~min}$. The light intensity is sampled at $10 \mathrm{~Hz}$, and the resulting data are wirelessly transmitted to a host computer by the ZigBee wireless network protocol within an operating range of 
approximately $20 \mathrm{~m}$. The system enables simultaneous measurement of up to 20 individuals.

Unlike generally used commercial continuous-wave fNIRS devices, our fNIRS device uses only a single wavelength that is at the vicinity of an isosbestic point of oxygenated and deoxygenated hemoglobin (Roggan et al., 1999). It thus estimates the concentration change of total hemoglobin by the modified Beer-Lambert law (Delpy et al., 1988; Scholkmann et al., 2014)

$$
-\ln \frac{I(t)}{I\left(t_{0}\right)}=\epsilon \Delta c_{H b}\langle L\rangle .
$$

Here, $I\left(t_{0}\right)$ and $I(t)$ denote the detected light intensity at the baseline $t_{0}$ and time $t$, respectively; $\Delta c_{\mathrm{Hb}}=c_{\mathrm{Hb}}(t)-c_{\mathrm{Hb}}\left(t_{0}\right)$ denotes the hemoglobin concentration change; $\epsilon$ is the extinction coefficient; $\langle L\rangle$ denotes the effective optical path length, which accounted for the differential path length factor. Thus, our fNIRS device gives signal $x=\Delta c_{H b}\langle L\rangle$ for each of the two S-D pairs with distances of $1.0 \mathrm{~cm}$ and $3.0 \mathrm{~cm}$, which we refer to as shallow signal $x_{\text {shallow }}$ and deep signal $x_{\text {deep }}$, respectively.

Based on involvement of the medial rostral prefrontal to frontopolar cortex (BA10) in processes that are essential for successful communication-such as mentalizing, understanding the beliefs, desires, and goals of others; and multi-task coordination (Amodio and Frith, 2006; Gilbert et al., 2006; Stephens et al., 2010)—we measured the neural activities of the frontopolar region to verify the hypothesized INS increase by the 
cooperative communication. The center of measurement optode component was positioned at the center between FP1 and FP2 according to the international 1020 system for electroencephalography electrode placement, which covered the rostral limit of superior frontal gyrus (Homan et al., 1987). The light source was placed on the left-hand side, the light detector with a $3-\mathrm{cm}$ distance from the source was on the right-hand side, and the light detector with 1-cm distance from the source was between them. Makeup on the measurement position was wiped off, and hair under the sensors were brushed away to ensure good optode-skin contact. The optode component was covered by black rubber to shield it from external lights.

\subsection{Artifact reduction methods for NIRS data}

Previous studies evaluated the effectiveness of various artifact reduction methods for better detection of task-evoked responses (Brigadoi et al., 2014; Cooper et al., 2012; Robertson et al., 2010). Among those methods, we focused on two methods that were reported to be quite effective for evoked-response studies: dual S-D regression and wavelet-based artifact reduction.

\subsubsection{Dual source-detector regression}

The dual or multiple S-D regression method uses the short distance S-D pairs in addition to the channels for brain blood-flow measurement with the usual S-D distance, which is typically $3 \mathrm{~cm}$ for adult subjects (Luu and Chau, 2009; Saager and Berger, 
2005; Saager et al., 2011; Toronov et al., 2001b). The short S-D distance (here $1 \mathrm{~cm}$ ) channel captures the shallow blood-flow signal, which is dominated by systemic components in the superficial layers of the sculp. The deep signal from the usual S-D distance $(3 \mathrm{~cm})$ channel contains both of the superficial and cortical (neural) blood-flow components. The dual S-D regression method formulates the deep signal as a linear combination of the shallow and neural signals; i.e.,

$$
x_{\text {deep }}=a_{0}+a_{1} x_{\text {shallow }}+x_{\text {neural }} \text {, }
$$

and it estimates coefficients $a_{0}$ and $a_{1}$ by the ordinary least squares method over the entire time course to best-fit the $x_{\text {shallow }}$ to $x_{\text {deep }}$, which is under the assumption that the shallow and neural signals are temporally uncorrelated. Thus, the neural signal is estimated as the residual component $x_{\text {neural }}=x_{\text {deep }}-\left(a_{0}+a_{1} x_{\text {shallow }}\right)$.

\subsubsection{Wavelet-based motion artifact reduction}

Molavi and Dumont (Molavi and Dumont, 2012) developed the original WBMR method. In this study, as an implementation of WBMR, we used an hmrMotionCorrectWavelet.m function implemented in the HomER2 fNIRS data analysis package (Huppert et al., 2009). This function is a slightly modified version from the original method and uses a ratio to the interquartile range instead of variance estimation based on the Gaussian distribution assumption for the detection of outlier wavelet coefficients. More specifically, the method calculates discrete wavelet 
coefficients $\left\{w_{j k}\right\}$ at each scale $j$, and it determines their lower quartile $Q_{1}=$ $\mathrm{CDF}^{-1}(0.25)$, upper quartile $Q_{3}=\mathrm{CDF}^{-1}(0.75)$, and the interquartile range $\mathrm{IQR}=Q_{3}-Q_{1}$ (here CDF represents the cumulative distribution function). Then, it identifies the outlier coefficients as those that are smaller than $Q_{1}-C_{\mathrm{iqr}} \times \mathrm{IQR}$ or larger than $Q_{3}+C_{\mathrm{iqr}} \times \mathrm{IQR}$, and replaces them by 0 , assuming these outliers account for the motion artifacts. Finally, it applies the inverse discrete wavelet transform on the modified coefficients and reconstructs the signal. The threshold parameter, $C_{\mathrm{iqr}}$, determines the method's sensitivity to outliers, with lower values corresponding to more stringent criteria in exchange for the higher risk of losing true signal variance. In this study, we compared the settings of $C_{\text {iqr }}=\operatorname{Inf}$ (i.e., no WBMR), 5.0, 4.0, 3.0, 2.0, 1.5, and 1.0.

Supplementary Figure S1 shows an example of how the WBMR with the different threshold parameters reduces the spike-like artifactual components from a deep signal.

\subsubsection{Flow of preprocessing}

The flow of NIRS data preprocessing is described as follows. Firstly, the third-order polynomial detrending was applied to remove possible drift components. Secondly, WBMR was applied with different values for the thresholding parameter $C_{\mathrm{iqr}}$. These processes were applied to both the deep and shallow signals from the $3-\mathrm{cm}$ and 1-cm S-D pairs. Finally, the dual S-D regression was applied to the deep and shallow 
signals, resulting in the neural signal. We situated the dual S-D regression at the end of the preprocessing flow because better regression fitting between deep and shallow signals is expected after removing trend and spike components.

\subsection{Analysis of interpersonal neural synchronization}

\subsubsection{Wavelet transform and wavelet transform coherence}

As described in the Introduction, naturalistic communication involving multiple individuals, which is herein addressed, is a highly dynamic process (Konvalinka and Roepstorff, 2012). This leads to the expectation that the INS occurrence during the communication will be fluctuating and transient with no clear directionality. To capture such transient synchronization, we used the wavelet transform coherence (WTC) (Grinsted et al., 2004; Torrence and Compo, 1998), as was done in previous fNIRS hyperscanning studies (Cui et al., 2012; Dommer et al., 2012; Holper et al., 2012; Jiang et al., 2015; Jiang et al., 2012).

After the preprocessing detailed above, the data of all pairs in each group were subjected to the WTC analysis using the cross wavelet and wavelet coherence toolbox for MATLAB (Grinsted et al., 2004). To summarize the procedure, firstly for each signal $x(n)$, its continuous wavelet transform $\mathrm{W}^{\mathrm{X}}(n, s)=\sqrt{\frac{\Delta t}{s}} \sum_{n^{\prime}=1}^{N} x_{n^{\prime}} \psi_{0}\left[\left(n^{\prime}-\right.\right.$ n) $\left.\frac{\Delta t}{s}\right]$ was calculated. Here $n$ is a time, $s$ is a wavelet scale, $\Delta t$ is the sampling time step size (=0.1 s), and $N$ is the length of the time series. For the wavelet function, we 
used the Morlet wavelet $\psi_{0}(\eta)=\pi^{-\frac{1}{4}} e^{i \omega_{0} \eta} e^{-\frac{1}{2} \eta^{2}}$ with the parameter $\omega_{0}=6$. Then, for the pair of signals $x(n)$ and $y(n)$, the cross-wavelet transform $\mathrm{W}^{\mathrm{XY}}(n, s)=$ $W^{X}(n, s) W^{Y *}(n, s)$ was calculated (the asterisk denotes complex conjugation). The wavelet transform coherence of $x(n)$ and $y(n)$ was given by

$$
R^{2}(n, s)=\frac{\left|\left\langle s^{-1} W^{X Y}(n, s)\right\rangle\right|^{2}}{\left|\left\langle s^{-1} W^{X}(n, s)\right\rangle\right|^{2}\left|\left\langle s^{-1} W^{Y}(n, s)\right\rangle\right|^{2}}
$$

Here the brackets $\langle\cdot\rangle$ denote a smoothing operation in time and scale [see (Chang and Glover, 2010; Grinsted et al., 2004)]. For these calculations, the number of scales per octave was set to 12 .

When characterizing a signal in terms of oscillatory components, it is more appropriate and general to express them in Fourier periods (inverse of Fourier frequencies) than in wavelet scales. For the Morlet wavelet, the Fourier period $\lambda$ is given by the corresponding scale $s$ multiplied by the Fourier factor of $\frac{4 \pi}{\omega_{0}+\sqrt{2+\omega_{0}^{2}}}$. With $\omega_{0}=6$, the period is approximately equal to the corresponding scale $(\lambda \sim 1.03 s)$ (Torrence and Compo, 1998).

At the beginning and end of the sessions, and at the boundaries between the Communicating and Independent blocks, edge-effects and the influence from the adjacent blocks contaminate the estimates of wavelet coefficients and WTC. Therefore, we determined the cone of influence (COI) for each of the time borders, and excluded the data in COI from the statistical analysis explained below. COI is defined at each 
scale as the time range in which the influence from the discontinuity or from the other block across the border on the wavelet power does not drop by a factor $e^{-2}$; thus, the contamination is not negligible. For the Morlet wavelet, COI for a time border point $n$ and scale $s$ is determined as $\operatorname{COI}(n, s)=\left\{n^{\prime}|| n-n^{\prime} \mid \leq \sqrt{2} s\right\}$ (Torrence and Compo, 1998).

Figure 2 exemplifies wavelet transform coherence of neural, deep, and shallow signals of two interacting pairs of participants, with COIs for the Communicating and Independent blocks depicted. 

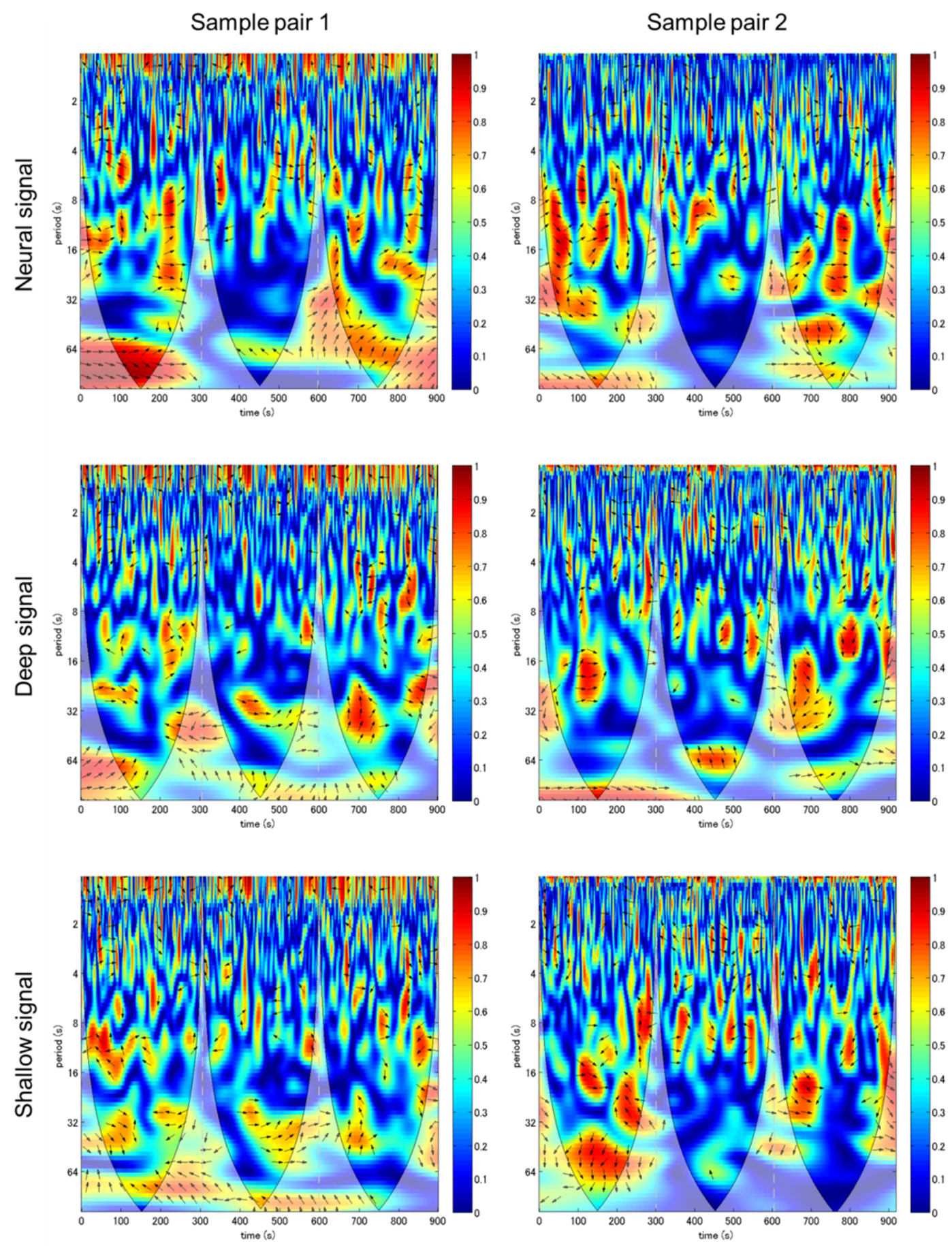

Figure 2. Illustrative examples of wavelet transform coherence (WTC) of neural (top), deep (middle), and shallow (bottom) signals from two interacting pairs of participants (left and right panels, respectively) from different groups. In each 
panel, vertical gray dashed lines indicate boundaries between Communicating (left and right) and Independent (middle) blocks. The faded color areas bordered by spindle-like curves indicate the cones of influence (COIs), which were determined for each block and the data points in them were excluded from the time-averaging of WTC in order to make the contaminating influence from the adjacent blocks and edge effects negligible (see main text). In the areas with high coherence values, arrows indicate phase angle of the paired signals, with right and left directions correspond to in-phase (0) and anti-phase ( $\pi)$, respectively.

\subsubsection{Temporal correlation of WTC between signal sources}

Furthermore, to delineate the source of conditional effects on the synchronization difference observed in the shallow signal (from the 1-cm S-D separation) and the neural signal (obtained by regressing out the shallow signal from the deep signal from the $3-\mathrm{cm}$ S-D separation), we performed correlation analysis of the WTC $R^{2}$ time series for each period. A high correlation reflects simultaneous increases and decreases of the interpersonal synchronization, which implies that the source of the transient interpersonal synchronization is shared between the two signals.

\subsubsection{Statistical analysis to compare conditions}

To investigate the effects of communication for INS and interpersonal synchronization in other signals, at the population level, we statistically tested the 
difference in the time-averaged WTC values for each signal type and their temporal correlations between Communicating and Independent conditions at each period in [1, 100] s (or $0.011 \mathrm{~Hz}$ ). The period range was chosen to cover virtually all frequency ranges investigated in previous INS studies and to exclude a higher frequency range ( $>$ $1 \mathrm{~Hz}$ ), which is unlikely to be of neural origin.

When considering a population-level statistical test on pairwise synchronization data in an experimental setting with more than two simultaneously interacting individuals, a new problem arises. That is, the pairwise synchronization in the group is not independent (e.g., when a pair, A B, is highly synchronized, and another pair, B C, is also highly synchronized, it is likely that pair A C would also be synchronized.) Thus, usual parametric tests that handle individual pairs as independent samples result in the risk of incorrectly inflated degrees of freedom. To resolve this problem, we used the framework of nonparametric permutation tests.

For each pair of participants in each group, the WTC was time-averaged at each period (scale) for respective Communicating and Independent conditions over all corresponding blocks in the two sessions, excluding data in the COIs for each block to avoid contaminating influence from the adjacent blocks and edge effects (see Section 2.5.1 and Figure 2). The between-condition difference (Communicating-Independent) was calculated for each pair of participants, and their sample z-score (mean divided by 
standard deviation) was calculated as the test statistics at each period. To test significant deviation from zero (i.e., a null hypothesis of no conditional difference), we generated 10,000 group-blocked random sign-flipping matrices in which signs of all pairs in each group were simultaneously flipped or unflipped, to maintain the pair-to-pair dependency structure. From the permutated data obtained by applying the sign-flipping matrices, the null distribution of the test statistics was derived. The significance of the original data's between-condition difference was tested (two-sided test) against that distribution. The between-condition differences in the temporal correlations of WTC between signal pairs were statistically tested in the same manner.

For the multiple testing for periods in the range of $[1,100] \mathrm{s}$, a false-discovery rate (FDR) adjustment was applied. The results with $q<0.05$ were regarded as significant. The statistical tests were conducted using the $\mathrm{R}$ software environment for statistical computing (R Development Core Team, 2008).

\subsection{Wavelet power spectral analysis of signals}

To elucidate how the preprocessing settings - especially the value of the WBMR thresholding parameter-affect the spectral characteristics of signals, we additionally examined the time-averaged wavelet power spectrum (WPS; also called the "global wavelet spectrum" (Torrence and Compo, 1998)) for each condition. We examined how the spectra differed between the signals and task conditions, depending on the values of 
the parameter. As discussed by Liu et al., when considering its energy contents in terms of time average, WPS as the squared wavelet coefficient is biased in favor of larger scales or equivalently lower scales (Liu et al., 2007). To rectify this bias, in evaluating WPS, the power was divided by each scale (Liu et al., 2007), resulting in the definition $\operatorname{WPS}^{X}(n, s)=\left|W^{X}(n, s)\right|^{2} / s$. The WPS was time-averaged at each scale, with the COI excluded, to produce the spectral power distribution curve.

We furthermore used the time-averaged WPS to observe timescale characteristics of utterance events. The utterance events for each person and for each group extracted at a 1-s time resolution from the video recording were encoded into a $1-\mathrm{Hz}$ sampling frequency of a binary time series with the value of 1 during the utterance and 0 during silence. The wavelet transform using the Morlet wavelet with the same parameters for the fNIRS signal analysis were applied to the binarized utterance event time series. The WPS was time-averaged for each scale for each Communicating block in the same way as explained above.

\section{Results}

\subsection{Characteristics of communication behavior}

In summarizing the utterance events during the Communication condition over all groups, sessions, and blocks, the mean $\quad$ SD of utterance frequency per group was 0.192

$0.057 \mathrm{~Hz}$, and the mean $\mathrm{SD}$ of utterance frequency per subject was $0.048 \quad 0.025$ 
Hz. Figures 3(a) and (c) show the distribution of group- and subject-wise inter-utterance intervals (IUIs). The median of the group- and subject-wise IUIs were $4 \mathrm{~s}$ and $13 \mathrm{~s}$, respectively. The tail of the group-wise IUI distribution corresponds to occasional stalling when no subject could produce an appropriate word to continue the word chain in the communication task. Figures 3(b) and (d) show the time-averaged WPS for the binarized utterance-silence time series during the Communication condition. The most characteristic timescales (periods for power peaks) for describing the group- and subject-wise utterance time series were approximately $3.9 \mathrm{~s}$ and $4.9 \mathrm{~s}$, respectively.
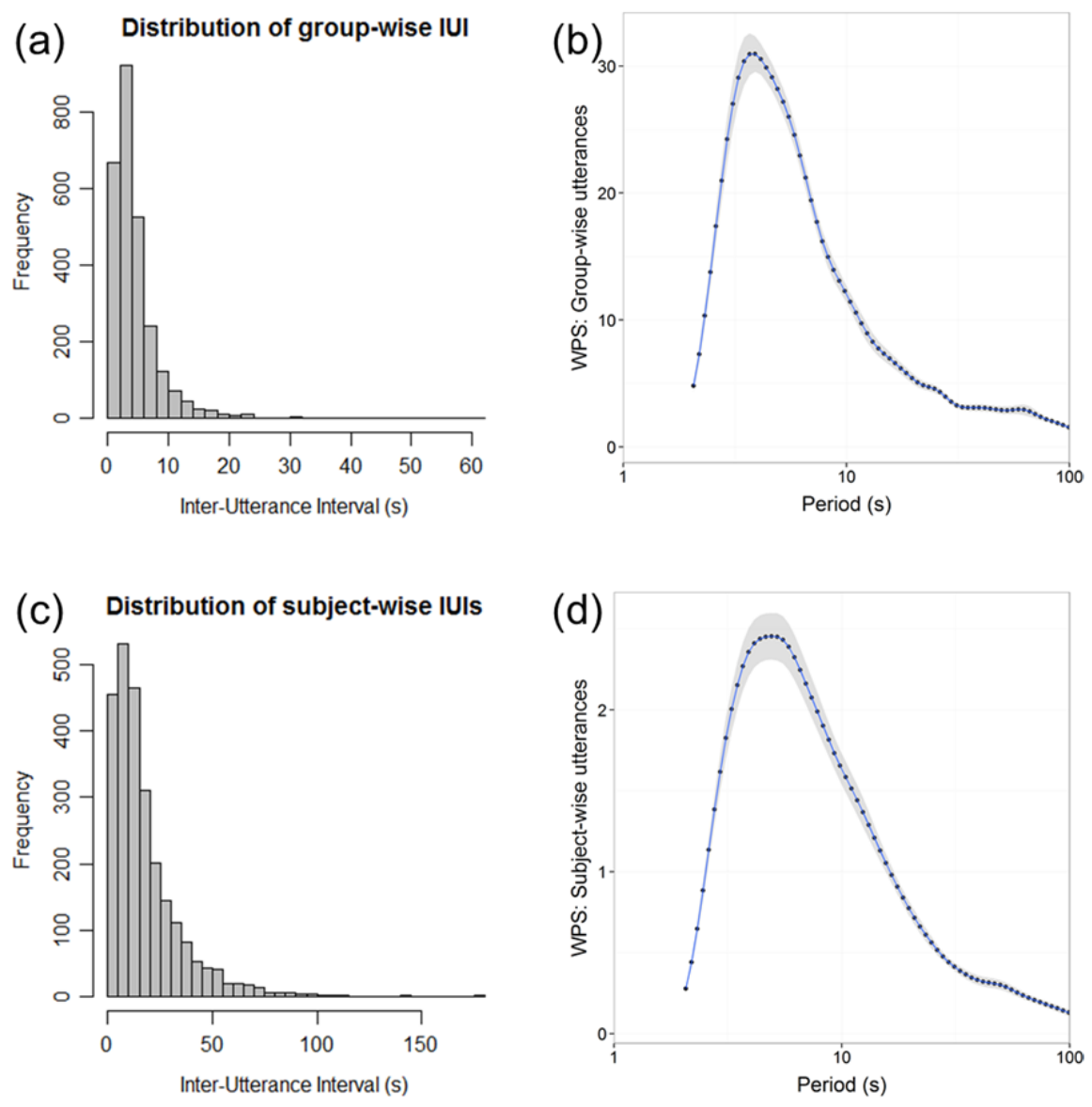

Figure 3. Temporal characteristics of utterance behavior during the 
Communicating block. (a) Distribution of group-wise inter-utterance interval; (b) time-averaged wavelet power spectrum (WPS) for the group-wise binarized utterance-silence time series; (c) distribution of subject-wise inter-utterance interval; (d) time-averaged WPS for the subject-wise binarized utterance-silence time series. For (b) and (d), the solid lines and shaded areas denote the mean and standard error of the mean (SEM) over all groups and participants, respectively.

\subsection{Enhanced INS during communication}

We evaluated the between-condition difference in INS in the neural signal, which was obtained by regressing out the preprocessed shallow signal from the deep signal. The time-averaged WTC was statistically compared between the conditions for each period in $[1,100]$ s. Figure $4(a)$ shows the comparison of the mean INS between Communicating and Independent blocks, with the thresholding parameter $C_{\mathrm{iqr}}=3.0$ for the WBMR method in the preprocessing flow. Significantly increased synchronization in the Communicating condition compared to the Independent condition was observed in the distributed timescales from short to long. (The period ranges with significant conditional differences were $1.752 .34 \mathrm{~s}, 4.957 .00 \mathrm{~s}, 13.216 .7$

s, 19.837 .4 s, $52.956 .0 \mathrm{~s}$, and $89.0100 \mathrm{~s}$; FDR-adjusted $q<0.05$.)

Incidentally, we tested whether the mixture of participants' acquaintance and 
gender (Table S1) induced significant difference on the effect of communication on the neural synchronization. Neither the acquaintance nor gender factor showed evident effects on the INS enhancement by communication (Figure S2(a) and S3(a)).

\subsection{Comparative interpersonal synchronization in the shallow and deep signals}

For comparison, we additionally evaluated the between-condition difference in the interpersonal synchronization in the deep and shallow signals (Figures 4(b) and (c)). The deep signal corresponds to the typical single $\mathrm{S}-\mathrm{D}$ distance $(3 \mathrm{~cm})$ measurement; the shallow signal reflects the superficial skin blood flow. Compared to the neural signal, the deep signal showed smaller interpersonal synchronization differences between the conditions (Figure 4(b)). The shallow signal furthermore showed interpersonal synchronization differences between the conditions over several distributed time scales, which were partly overlapped with the results of the neural and deep signals (Figure $4(c))$.
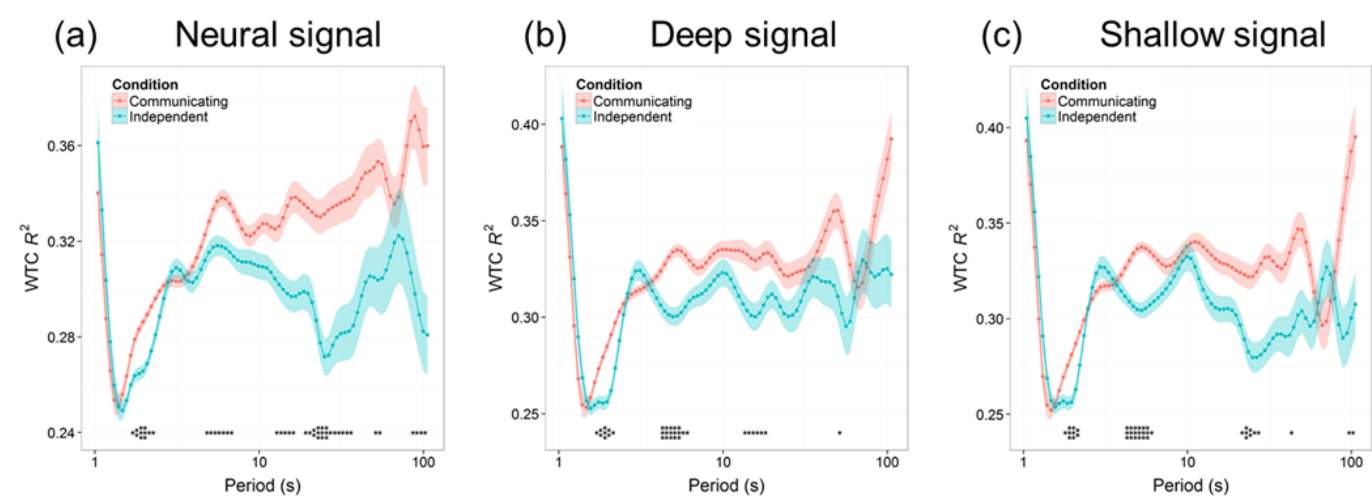

Figure 4. Comparison of interpersonal synchronization (INS) of the frontopolar signals between Communicating and Independent conditions, using $C_{\mathrm{iqr}}=\mathbf{3 . 0}$ 
for the discrete wavelet-based motion artifact reduction (WBMR) method in the preprocessing flow. (a) Time-averaged wavelet transform coherence (WTC) in the neural signal, which was obtained by regressing out the effect of the shallow $(1-\mathrm{cm}$ S-D pair) signal from the deep (3-cm S-D pair) signal; (b) time-averaged WTC in the deep signal, which corresponds to the typical single S-D distance measurement; (c) time-averaged WTC in the shallow signal, which is considered to be dominated by non-neuronal components. Solid lines show the mean of time-averaged WTC over all the interacting pairs of participants in all groups for each condition. Shaded areas show the standard error of the mean (SEM) over interacting pairs in all groups. Asterisks at the bottom indicate periods with significant between-condition differences; $* \mathbf{q}<.05, * * \mathbf{q}<.01, * * * \mathbf{q}<.001$ (FDR-adjusted). For reference, we also tested the interaction effects of participants' acquaintance and gender on the synchronization change by communication in the deep and shallow signals, finding no significant effects (Figure S2(b), (c) and S3(b), (c), respectively).

\subsection{Temporal correlation between shallow and neural synchronization}

The apparent similarity of conditional differences in interpersonal synchronization between the neural (Figure 4(a)), deep (Figure 4(b)), and shallow (Figure 4(c)) signals may invoke the concern that the observed enhanced INS effect was artifactually induced by head or facial motions, which could have been more frequent in Communicating 
conditions with overt utterances. The possibility also exists that the effect was caused by the systemic components shared in both the shallow and deep signals, rather than the neural activities in the frontopolar cortex. If these were the case, and the observed synchronization difference in the neural signal was merely propagated from the artifactual synchronization in the shallow signal, the timings of synchrony increase and decrease would be temporally correlated between the two signals. To investigate this possibility, we evaluated the temporal correlation between the time courses of interpersonal synchronization in different signal sources and compared them.

Figure 5 shows temporal correlations of WTC time series between the neural, deep, and shallow signals for each period in the range of periods $[1,100]$ s. The WTC temporal correlations between the deep and shallow signals were moderate to high over most periods. In addition, significant differences between the conditions were also observed in most periods (Figure 5(a)). In contrast, the temporal correlations of WTC between the neural and shallow signals were relatively low and closer to zero for larger periods. Differences between the conditions were also not significant for larger periods (Figure 5(b)). Finally, the temporal correlations of WTC between the deep and neural signals were relatively low compared to the deep shallow pair, with no significant conditional differences over most periods (Figure 5(c)). 

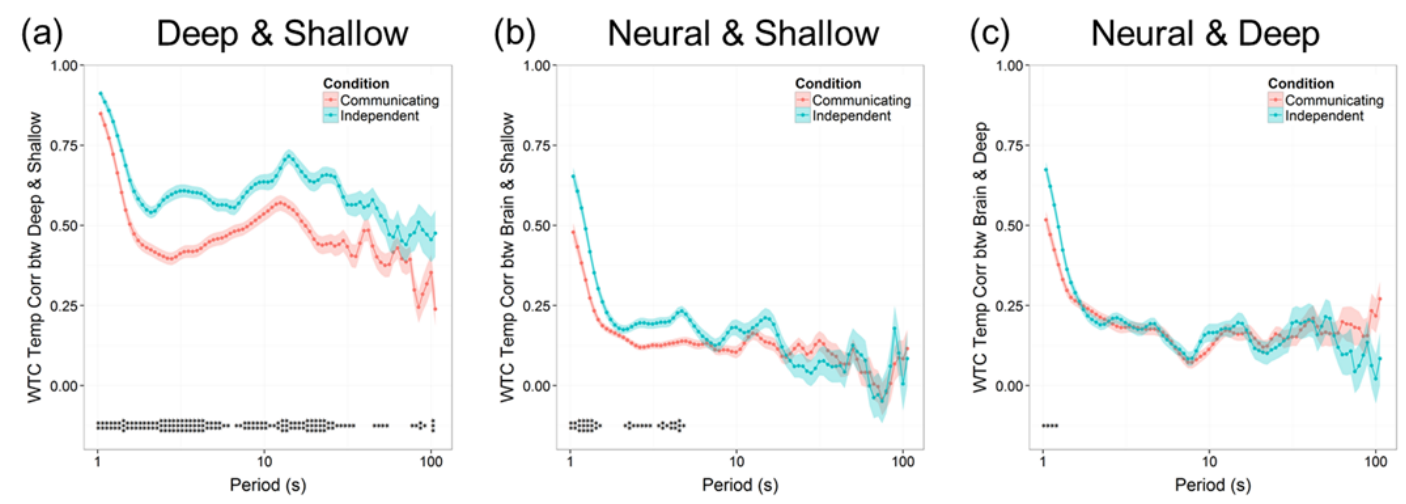

Figure 5. Temporal correlation of interpersonal synchronization between the different signal sources. Temporal correlations of the WTC time series between (a) deep and shallow signals, (b) neural and shallow signals, and (c) neural and deep signals were respectively calculated for Communicating and Independent conditions. $C_{\mathrm{iqr}}=3.0$ was used for the WBMR method in the preprocessing of the signals. Solid lines and shaded areas respectively show the mean and SEM of the temporal correlation averaged over all the interacting pairs of participants in all groups for each condition. Asterisks at the bottom indicates periods with significant between-condition difference; ${ }^{*} \mathrm{q}<.05,{ }^{* *} \mathrm{q}<.01$, ${ }^{* * *} \mathrm{q}<.001$ (FDR-adjusted).

\subsection{Effects of wavelet-based artifact reduction}

We evaluated the parameter-dependent effects of the discrete wavelet-based motion artifact reduction (WBMR) method on the detection of communication-enhanced INS. Extending Figure 4, Figure 6 shows the comparison of mean interpersonal synchrony in neural, deep, and shallow signals between 
Communicating and Independent blocks, with the different WBMR thresholding parameter values $C_{\mathrm{iqr}}=\operatorname{Inf}($ i.e., no WBMR), 5.0, 4.0, 3.0, 2.0, 1.5, and 1.0.

For reference, supplementary Figure S4 extends Figure 5 and shows the temporal correlations of the WTC time series between the neural, deep, and shallow signals with the WBMR thresholding parameter values $C_{\text {iqr }}=\operatorname{Inf}($ i.e., no WBMR), 5.0, 4.0, 3.0, 2.0, 1.5, and 1.0. 


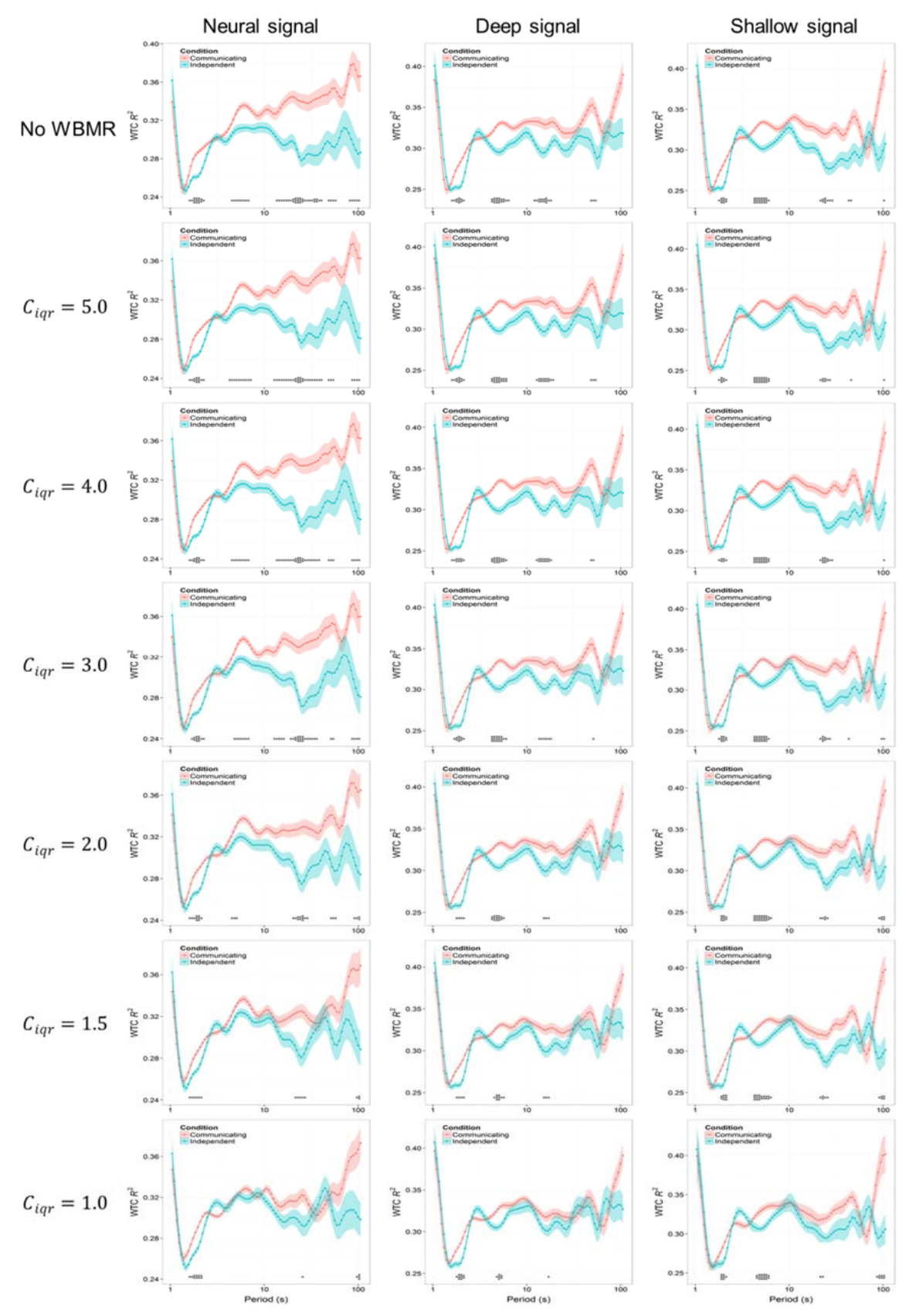

Figure 6. Comparison of interpersonal synchronization of the frontopolar neural, deep, and shallow signals between Communicating and Independent blocks, using different parameter values $C_{\mathrm{iqr}}=\operatorname{Inf}($ no $\mathrm{WBMR}), 5.0,4.0,3.0,2.0,1.5$, and 1.0 for the discrete wavelet-based motion artifact reduction (WBMR) method. Solid lines and shaded areas show the mean and SEM of time-averaged WTC over all 
the interacting pairs of participants in all groups for each condition. Asterisks at the bottom indicates periods with significant between-condition differences; ${ }^{*} \mathrm{q}$ $<.05, * * \mathbf{q}<.01, * * * \mathbf{q}<.001$ (FDR-adjusted).

To investigate the origin of such parameter dependence, we additionally examined WPS changes of the neural, deep, and shallow signals for different thresholding parameters of the WBMR method. Figures 7(a) (f) respectively show the time-averaged WPS of neural, deep, and shallow signals for Communicating and Independent blocks, with WBMR thresholding parameter values $C_{\text {iqr }}=\operatorname{Inf}$ (i.e., no WBMR), 5.0, 4.0, 3.0, 2.0, 1.5, and 1.0. Transforming these data, Figures $7(\mathrm{~g})$ (l) show the ratio of time-averaged wavelet power by $C_{\mathrm{iqr}}=5.0,4.0,3.0,2.0,1.5$, and 1.0 with respect to the time-averaged wavelet power by $C_{\mathrm{iqr}}=\operatorname{Inf}($ no WBMR) at each period for each signal and each block type. Here, we emphasize two main points to be discussed later. Firstly, introduction of the wavelet-based method, even with a tolerant threshold $C_{\mathrm{iqr}}=5.0$, induced noticeable signal power reduction in shorter periods between $1 \mathrm{~s}$ and $10 \mathrm{~s}$ for all signals (evident in Figures 7(g) (1), with the trough at $23 \mathrm{~s}$ ). In the raw time series, this reflects reduction of spike artifacts, as exemplified in Supplementary Figure S1. Secondly, focusing on the parameter-dependent change in neural WPS in the Independent condition (Figure $7(\mathrm{j})$ ), power in a longer timescale (period $>10 \mathrm{~s})$ was 
preserved for $C_{\mathrm{iqr}} \geq 3.0$; however, it notably decreased for $C_{\mathrm{iqr}} \leq 2.0$.

Incidentally, comparison of the WPS furthermore showed power peaks around period $=10 \mathrm{~s}$ shared in the deep and shallow signals specifically for the Communicating condition (Figures 7(b) (c)); however, the peak was considerably reduced in the neural signal (Figure 7(a)). 

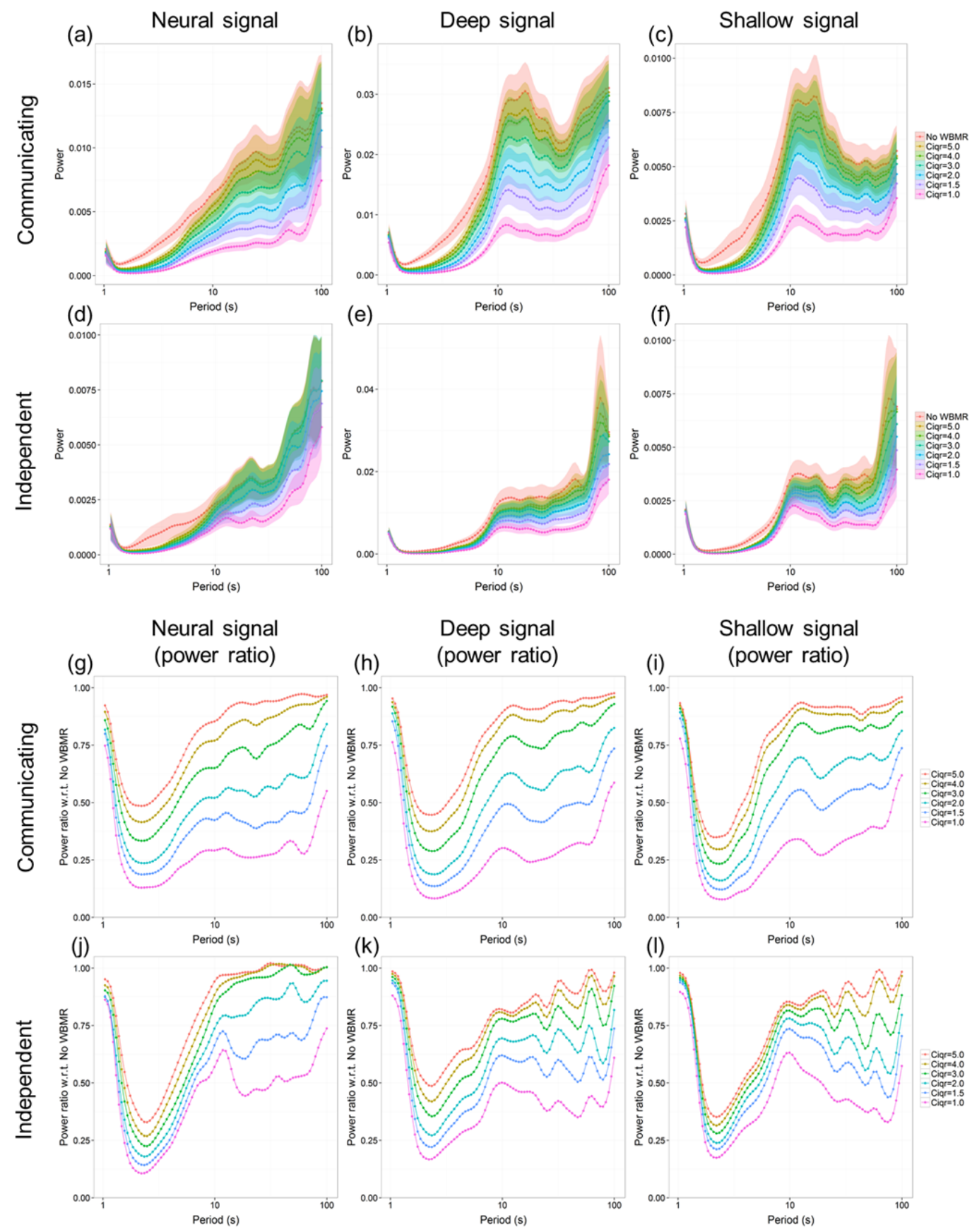

Figure 7. (a) (f) Comparison of time-averaged wavelet power spectra of the frontopolar neural, deep, and shallow signals for each of the Communicating and Independent conditions using different parameter values $C_{\mathrm{iqr}}=$ Inf (no WBMR), 5.0, 4.0, 3.0, 2.0, 1.5, and 1.0 for the WBMR method. Solid lines and shaded areas 
show the mean and SEM of the time-averaged WPS over all participants in all groups. (g) (l) Ratio of the time-averaged wavelet power for $C_{\text {iqr }}=5.0,4.0,3.0$, 2.0, 1.5, and 1.0 with respect to $C_{\mathrm{iqr}}=\operatorname{Inf}($ no WBMR), which demonstrates how stricter thresholds of the WBMR method affect at each timescale the signal power compared to when the method is not applied.

\section{Discussion}

\subsection{Interpersonal prefrontal synchrony in unstructured group verbal} communication

The current study investigated the neural synchrony between individuals engaged in natural communication. As expected from its role in social interactions, such as mentalizing, we observed an increase of the frontopolar INS during communication compared to during silent thinking. The results of fNIRS studies to date have been indecisive on the enhancing effect of cooperative communication on INS in the frontopolar region. Some studies showed a significant relationship between communication and medial prefrontal INS (Funane et al., 2011); some showed the effect for a limited population, i.e., female-male pairs (Cheng et al., 2015), and some showed the relationship between aspects of communication and INS in other regions, such as the inferior frontal cortex (Jiang et al., 2012) and temporo-parietal junction (Jiang et al., 2015), but not in the frontopolar region. Thus, the present study may be the first to 
provide clear evidence that natural/unstructured and cooperative verbal communication between more than two individuals can induce enhanced INS in the frontopolar region. Several possible reasons exist for our success in detecting communication-enhanced INS in the frontopolar region. Firstly, the analysis in the finer timescale bins may have helped in capturing enhanced INS, which could have been missed when one averaged an INS measure over a gross range of periods. Secondly, and perhaps more significantly, removal of systemic noise by the dual S-D regression was effective in improving the detection of INS enhancement, as is demonstrated by the comparison of results from the neural versus deep signals in Figures 4 and 6, as well as by the temporal segregation of the INS occurrences from artifactual synchronization in Figure 5. These points are detailed in the following. Among other possible reasons, the nature of the communication task may have provided sufficient room for enhancement of INS because it would have involved social (guessing partners' thoughts, intentions, and knowledge), emotional (joy of success and stress in stasis), cognitive (retrieval of memory and creativity) components, and large swings between these different "modes." Moreover, in our analysis, the baseline was silent thinking with no communication; thus, the enhancement of INS could have been easier to detect compared to other studies aiming for the relationship of INS with more delicate aspects of communication.

Incidentally, we also investigated whether the participants' acquaintance or gender 
affected the INS enhancement by communication, and observed no evident effects for either factor. However, these results are not conclusive because such effects were not the focus of this study and the samples were not well-balanced on these attributes (Table S1). Future studies pursuing on these issues should employ a well-balanced design, as has been done in (Cheng et al., 2015).

\subsection{INS changes in different timescales possibly involves different mechanisms}

We observed the INS increase by communication in widely distributed timescales in the examined period range of $[1,100] \mathrm{s}$ (Figure 4). Notably, the INS enhancement was not limited to the timescale that was directly connected to utterance behavior (Figures 3(b) and (d)). As suggested in Figure 5, and as will be discussed further below, the enhancing effect at a longer timescale was most likely of neural origin. This suggests the merit of looking into effects on INS, not only in a directly task-related band of periods, but in a wider range of timescales.

Resting-state fMRI studies show that low-frequency oscillations (LFO), typically in $[0.01,0.1] \mathrm{Hz}$, reflect within-brain neuronal synchronization more reliably than higher frequencies do (Achard et al., 2006; Zuo et al., 2010), which increases the credibility of the observed communication-enhanced INS in that timescale being of neuronal origin. Moreover, sub-scales in LFO have been shown to contribute differently to regions, functional networks, and psychological/psychiatric 
characteristics (Baria et al., 2011; Nakao et al., 2013; Sasai et al., 2011; Wei et al., 2014; Yu et al., 2014). These previous findings seem to be in accordance with our result of communication-enhanced INS at discontinuous sub-scales in LFO suggesting the existence of different mechanisms underlying the INS at these sub-scales in LFO.

On the other hand, we also found significant between-condition difference in INS at higher-frequency $(>0.1 \mathrm{~Hz}$ or period $<10 \mathrm{~s})$ sub-scales. Although the temporal correlations of interpersonal synchronization (WTC dynamics) between the neural and shallow signals in the higher-frequency bands were higher than in the LFO range (Figure 5(b)), the correlation values were low compared to those between the deep and shallow signals (Figure 5(a)). (Additional discussion on the temporal correlation of dynamic coherence between the neural and shallow signals is given in the next subsection.) Considering recent fMRI studies that indicate the neural relevance and functional contribution of higher-frequency fluctuations in BOLD signals (Chen and Glover, 2015; Gohel and Biswal, 2015), we cannot exclude the possibility that at least part of the communication-enhanced interpersonal synchronization observed in the higher-frequency sub-scales was of neural origin.

To further clarify the functional meaning of different timescales, we propose that future studies likewise analyze the effects of communication factors on INS in fine 
timescale bins, especially when using realistic communication settings where various timescales can interact at the behavioral level.

\subsection{Regression of shallow signal improves detection of INS}

We found that dual S-D regression made a significant improvement in detecting INS change by communication. Effectiveness of the method and importance of excluding the skin blood flow component was supported not only by average improvement in detection of significant between-condition differences in the time-averaged synchrony (Figures 4 and 6), but also by improved temporal segregation of the INS occurrences from the occurrence of artifactual synchronization. This latter aspect was shown in the drastically reduced temporal correlation of dynamic coherence between the neural and shallow signals compared to the deep and shallow signals (Figures 5 and S4). The low temporal correlation between the neural and shallow signals (Figure 5(b); especially for period $>10 \mathrm{~s}$ ) indicate that the enhancement of synchrony observed in the neural and shallow signals occurred at nearly independent timings, despite their apparent similarity of the time-averaged WTC profiles (Figure 4(a) and (c)). This alleviates the concern that the observed enhanced INS effect may have been artifactually induced by head or facial motions or the systemic components shared in both the shallow and deep signals, rather than the neural activities.

In comparison, the higher WTC temporal correlations between the deep and 
shallow signals (Figure 5(a)) indicate similarity in timings of synchrony increase and decrease between these signals, thus suggesting the artifact susceptibility of INS evaluation in the frontopolar region using the usual fNIRS measurement without compensating for shallow signal components. It has been implicated that a large part of the NIRS signal in the frontopolar region can originate in skin blood flow controlled by the autonomic nerve system (Haeussinger et al., 2014; Takahashi et al., 2011). Therefore, it is possible that contamination from the skin blood flow obstructed the detection of communication-related changes in the frontopolar INS in previous studies.

Interestingly, for the temporal correlation between the deep and shallow signals (Figure 5(a)), we observed a higher correlation during the Independent block as opposed to the Communicating blocks. This can be understood in terms of the influence from physiological components. That is, during silent thinking in the Independent block, physiological processes, such as cardiac pulsation, respiration, and Mayer waves, could have been stable and thus more likely to incidentally synchronize in their dominant frequency bands (around 1, 0.25, and $0.1 \mathrm{~Hz}$, respectively) (Katura et al., 2006; Matthews et al., 2008; Zhang et al., 2010). On the other hand, communication may have made those physiological processes more dynamic and thus disrupted the incidental synchronization.

There was a small but positive correlation between the neural and shallow signals, 
especially in the period $<10 \mathrm{~s}$ (Figure $5(\mathrm{~b})$ ). This may reflect the effect of autonomic nervous components that remained even after the dual S-D regression, or the causal coupling between the frontopolar activity and the autonomic nervous system. A previous study observed increased correlation between the BOLD signals between the skin tissue and gray matter at the frontopolar region (Haeussinger et al., 2014), suggesting the possibility of the latter case.

Previous fNIRS hyperscanning studies have reported interaction-enhanced INS in regions other than the frontal pole, such as the superior frontal cortex (Cui et al., 2012), inferior frontal cortex (Jiang et al., 2012), premotor cortex (Holper et al., 2012; Jiang et al., 2012), and temporo-parietal junction (Jiang et al., 2015). It is worth exploring in future studies how the skin blood flow removal by dual S-D regression or other methods can improve and extend those results.

On a somewhat related note, previous studies have suggested that superficial physiological signals tend to be spatially more global and homogeneous than the neural signals (Kohno et al., 2007; Zhang et al., 2005) [however, see also (Gagnon et al., 2012a; Kirilina et al., 2012)]. As a possibility which is beyond the scope of this study, influence from skin blood flow on INS may also be expressed globally, and thus can be more easily detected by using fNIRS instruments with many measurement channels. 


\subsection{Effects of wavelet-based motion reduction on INS analysis}

We observed that the WBMR method with intense removal of outliers (i.e., a lower

threshold parameter $C_{i q r}$ ) smeared the condition differences in INS (Figure 6). The comparison of the neural signal WPS between the different threshold parameters indicated the concurrence of the degenerated sensitivity to INS differences and the signal power loss over wide timescales with weight on longer periods (Figure 7). Specifically for neural WPS in the Independent condition (Figure 7(j)), the power in LFO was preserved for thresholding parameters $C_{\mathrm{iqr}} \geq 3.0$ and noticeably decreased for $C_{\mathrm{iqr}} \leq 2.0$. Considering that the Independent condition was free from motion and physiological artifacts caused by verbal communication, the result suggests that, for this dataset, the parameter values of $C_{\mathrm{iqr}}=3.0$ or higher maintained slow $(<0.1 \mathrm{~Hz})$ fluctuation of neural origin, while $C_{\mathrm{iqr}}=2.0$ or lower values may have risked gradual loss of the slow neural fluctuation.

These results suggest the possibility that a too-intense WBMR removes neural fluctuation components that can be better removed as noise in the case of targeting task-evoked responses but is essential for INS. Resting state imaging studies suggest that fluctuations of neural activity contributing inner-brain functional connectivity consist of not only stationary, but also sporadic components, which possibly originate from the neuronal avalanche that shows power-law size and timescale distributions 
(Allen et al., 2014; He et al., 2010; Hutchison et al., 2013; Liu and Duyn, 2013; Palva et al., 2013; Tagliazucchi et al., 2012). Although this is a speculation, our results suggest a possibility that sporadic neural events with large timescales (lifetimes or intervals) or amplitude also contribute to INS, and the intense WBMR filtered out as outliers such components in longer timescales.

On the other hand, against our expectations, reduction of spike artifacts by WBMR with a tolerant thresholding parameter when compared with no use of WBMR did not produce an evident improvement in the detection of communication-enhanced INS (Figure 6). A possible explanation of this result is the use of WTC as the dynamic synchrony measure. As shown in the definition of Eq. (1), WTC involves normalization in terms of amplitude within each virtual time-window defined by the wavelet; thus, it is not vulnerable to the existence of transient abnormally large amplitudes induced by spike artifacts. In comparison, in regression-based methods based on the assumption of the stationarity of signals, such as Granger causality and the multivariate autoregressive (MVAR) model, abnormalities of the outlier amplitude would be directly propagated to the estimate of coefficients and would thus have a greater impact on the population-level estimates and between-condition comparisons. Another possible explanation is that the motion artifacts are generally less influential for the estimation of interpersonal coherence, in comparison to when estimating within-individual 
inter-regional coherence, because occurrences of motions do not tightly coupled between individuals. This could have been the case especially in the unstructured verbal communication involving multiple individuals.

However, utility of the WBMR method could also depend on the experimental setting. The task in this study was verbal communication in the sitting position. Participants were instructed to assume a relaxed posture and maintain it as much as possible. It is likely that, in more dynamic communication settings involving bodily interactions, such as walking and gestures, an intense threshold parameter for artifacts can benefit in improving the detection of INS. A dyadic communication with fixed roles may induce tighter interpersonal coupling of motions, also leading to higher importance of motion artifact reduction.

In summary, our results call for caution in optimal parameter setting for each data and the choice of the INS measure when using the WBMR method in preprocessing.

\subsection{Limitations}

As described in Section 2, the fNIRS device we used in this study employs only a single wavelength of light, resulting in estimation of the change in only totalHb, which corresponds to the sum of changes in oxyHb and deoxyHb. Moreover, it is largely dominated by the oxyHb change. Thus, we cannot exclude the possibility of additional useful information that deoxyHb can provide to characterize inter-individual neural 
synchrony. However, previous fNIRS studies on two-person neural synchrony have reported synchrony in terms of either oxyHb (Cui et al., 2012; Funane et al., 2011; Jiang et al., 2015; Jiang et al., 2012) or totalHb changes (Dommer et al., 2012; Holper et al., 2012). Gagnon et al. suggested that totalHb change is less sensitive to pial vein contamination and thus may provide better spatial specificity than oxyHb or deoxyHb changes (Gagnon et al., 2012b). Thus, we infer that our analysis based on the totalHb measurement is sufficiently sensitive to detect the primary components of the INS.

A related issue we could not investigate in this study is the effectiveness of the correlation-based signal improvement (CBSI) method (Cui et al., 2010), which is based on the observation that oxyHb and deoxyHb signals are usually negatively correlated, but that head motion induces a positive correlation. Effectiveness of the CBSI method, along with other artifact reduction methods used in this study, has been evaluated for detection of classical task-evoked activation (Brigadoi et al., 2014). Such a comparative evaluation of effectiveness of the CBSI method in capturing INS is to be conducted in a future study.

Using the dual S-D regression, our results showed the importance of appropriately dealing with the influence of shallow signal in order to dissociate prefrontal INS from artefactual physiological synchronization. However, the dual S-D regression is not necessarily the best method because it is based on assumptions which are not always 
met, such as that the influence weight of superficial signal is constant over time (stationarity), or that the superficial and neural signals are temporally not correlated (orthogonality). Regarding the former assumption, several studies proposed usage of adaptive filtering or other approaches to account for nonstationarity of the influence from shallow signals (Gagnon et al., 2011; Izzetoglu et al., 2010; Zhang et al., 2009). To alleviate the risk of over-subtraction of neural signals caused by the latter assumption (Kirilina et al., 2012), Funane et al. developed a method combining multi-distance optodes and independent component analysis (MD-ICA) to allow the inclusion of "shared components" between the shallow and neural signals (Funane et al., 2014). It will be worth exploring the effectiveness of these different utilizations of shallow signal measurements for INS evaluation, and also developing a new method that is free from both the above assumptions.

Among various confounding factors for fNIRS measurement, expected importance of managing changes in respiration may warrant a special note. It has been shown that modulation on respiration affects both the deep and shallow signals (Obrig et al., 2000), making the removal of the confounding effect more difficult. Direct effect of respiration is of particular relevance when both the interaction task under study and the observed INS are in the frequency range of respiration, as has been the case in a study of cooperative singing/humming (Osaka et al., 2015). On the other hand, variations in 
respiration can affect the blood flow signals at the lower frequency band that overlaps LFO (Birn et al., 2008). Therefore, for fNIRS hyperscanning studies with interaction tasks involving respiration changes, respiration monitoring, response function estimation, and regressing out in the similar manner as for fMRI studies (Birn et al., 2006; Birn et al., 2008) can be useful.

\subsection{Outlook}

For the emerging research field of fNIRS hyperscanning in real-life collective interactions, an exciting future direction would be to investigate the functional contributions of INS to various communication quality measures or emergent social phenomena, such as mutual understanding, empathy, rapport, group flow, feeling of unity, formation and transition of roles and bonded subgroups. Jiang et al. conducted a pioneering work on this direction and showed that INS in the left temporo-parietal junction distinguish an emergent leadership in a group discussion task (Jiang et al., 2015). Although it was beyond the scope of this study, future research is warranted to investigate the possible contributions of frontopolar INS to such emergent properties in natural interactions.

In the broader context, to make the endeavor of real-life fNIRS hyperscanning successful, reliable and reproducible evidences, and methodological development to achieve that will become essential. In particular, from the results of the current study, it 
would be advisable for future fNIRS hyperscanning studies to analyze data as comprehensively as possible, keeping comparability over studies in mind.

\section{Conclusions}

Research of INS using fNIRS hyperscanning is a nascent field that calls for accumulation of findings and establishment of analytic standards. In this study, we conducted an fNIRS hyperscanning study while groups of four young adults were engaged in cooperative verbal communication. Our results (1) confirm that frontopolar INS is enhanced by unstructured communication, as expected from the region's roles in communication; (2) revealed the existence of timescale dependency in the INS modulation induced by communication, suggesting the merit of evaluating INS in fine timescale bins; and (3) determined that an additional measurement and removal of influence from skin blood flow engenders substantial improvement in sensitivity to capturing communication-enhanced INS and temporal segregation of INS changes from artifactual synchronization observed in physiological components. Moreover, it suggests that use of the WBMR method may require optimal parameter setting, which may differ from the optimal setting investigated for usual task-evoked response studies. Hopefully, the present study can provide a basis for the new field of massive hyperscanning studies during daily interactions, such as those in classrooms, meetings, sports, and theaters, to 
explore how collective neural synchrony can reflect the quality of the interactions.

\section{Acknowledgments}

We thank the staff of Hitachi Ltd. for the collaborative development of the fNIRS

device, as well as the participants and all our colleagues in IDAC, Tohoku University

for their support. This study was partially supported by System Development Program for Advanced Measurement and Analysis, Japan Science and Technology Agency (JST; http://www.jst.go.jp/), and also by MEXT/JSPS (http://www.jsps.go.jp/) KAKENHI Grant Numbers 23700137 and 15H01771.

\section{References}

Achard, S., Salvador, R., Whitcher, B., Suckling, J., Bullmore, E., 2006. A resilient, low-frequency, small-world human brain functional network with highly connected association cortical hubs. J Neurosci 26 (1), 63-72.

Allen, E. A., Damaraju, E., Plis, S. M., Erhardt, E. B., Eichele, T., Calhoun, V. D., 2014. Tracking whole-brain connectivity dynamics in the resting state. Cereb Cortex 24 (3), 663-676.

Amodio, D. M., Frith, C. D., 2006. Meeting of minds: the medial frontal cortex and social cognition. Nat Rev Neurosci 7 (4), 268-277.

Astolfi, L., Toppi, J., Fallani, F. D. V., Vecchiato, G., Salinari, S., Mattia, D., Cincotti, F., Babiloni, F., 2010. Neuroelectrical hyperscanning measures simultaneous brain activity in humans. Brain Topogr 23 (3), 243-256. 
Babiloni, F., Astolfi, L., 2014. Social neuroscience and hyperscanning techniques: past, present and future. Neurosci Biobehav Rev 44, 76-93.

Baria, A. T., Baliki, M. N., Parrish, T., Apkarian, A. V., 2011. Anatomical and functional assemblies of brain bold oscillations. J Neurosci 31 (21), 7910-7919.

Bilek, E., Ruf, M., Schäfer, A., Akdeniz, C., Calhoun, V. D., Schmahl, C., Demanuele, C., Tost, H., Kirsch, P., Meyer-Lindenberg, A., 2015. Information flow between interacting human brains: Identification, validation, and relationship to social expertise. Proc Natl Acad Sci U S A 112 (16), $5207-5212$.

Birn, R. M., Diamond, J. B., Smith, M. A., Bandettini, P. A., 2006. Separating respiratory-variation-related fluctuations from neuronal-activity-related fluctuations in fMRI. Neuroimage 31 (4), 1536-1548.

Birn, R. M., Smith, M. A., Jones, T. B., Bandettini, P. A., 2008. The respiration response function: the temporal dynamics of fMRI signal fluctuations related to changes in respiration. Neuroimage $40(2), 644-654$.

Brigadoi, S., Ceccherini, L., Cutini, S., Scarpa, F., Scatturin, P., Selb, J., Gagnon, L., Boas, D. A., Cooper, R. J., 2014. Motion artifacts in functional near-infrared spectroscopy: a comparison of motion correction techniques applied to real cognitive data. Neuroimage 85 Pt 1, 181-191.

Chang, C., Glover, G. H., 2010. Time-frequency dynamics of resting-state brain connectivity measured with fMRI. Neuroimage 50 (1), 81-98. 
Chen, J. E., Glover, G. H., 2015. Bold fractional contribution to resting-state functional connectivity above 0.1 hz. Neuroimage 107, 207-218.

Cheng, X., Li, X., Hu, Y., 2015. Synchronous brain activity during cooperative exchange depends on gender of partner: A fNIRS-based hyperscanning study. Hum Brain Mapp 36 (6), 2039-2048.

Cooper, R. J., Selb, J., Gagnon, L., Phillip, D., Schytz, H. W., Iversen, H. K., Ashina, M., Boas, D. A., 2012. A systematic comparison of motion artifact correction techniques for functional near-infrared spectroscopy. Front Neurosci 6, 147.

Cui, X., Bray, S., Reiss, A. L., 2010. Functional near infrared spectroscopy (NIRS) signal improvement based on negative correlation between oxygenated and deoxygenated hemoglobin dynamics. Neuroimage 49, 3039-3046.

Cui, X., Bryant, D. M., Reiss, A. L., 2012. NIRS-based hyperscanning reveals increased interpersonal coherence in superior frontal cortex during cooperation. Neuroimage 59 (3), 24302437.

De Vico Fallani, F., Nicosia, V., Sinatra, R., Astolfi, L., Cincotti, F., Mattia, D., Wilke, C., Doud, A., Latora, V., He, B., Babiloni, F., 2010. Defecting or not defecting: how to "read" human behavior during cooperative games by EEG measurements. PLoS One 5 (12), e14187.

Delpy, D. T., Cope, M., van der Zee, P., Arridge, S., Wray, S., Wyatt, J., 1988. Estimation of optical pathlength through tissue from direct time of flight measurement. Phys Med Biol 33 (12), 14331442. 
Dommer, L., Jäger, N., Scholkmann, F., Wolf, M., Holper, L., 2012. Between-brain coherence during joint n-back task performance: a two-person functional near-infrared spectroscopy study.

Behav Brain Res 234 (2), 212-222.

Duan, L., Dai, R.-N., Xiao, X., Sun, P.-P., Li, Z., Zhu, C.-Z., 2015. Cluster imaging of multi-brain networks (CIMBN): a general framework for hyperscanning and modeling a group of interacting brains. Front Neurosci 9, 267.

Dumas, G., Nadel, J., Soussignan, R., Martinerie, J., Garnero, L., 2010. Inter-brain synchronization during social interaction. PLoS One 5 (8), e12166.

Egetemeir, J., Stenneken, P., Koehler, S., Fallgatter, A. J., Herrmann, M. J., 2011. Exploring the neural basis of real-life joint action: Measuring brain activation during joint table setting with functional near-infrared spectroscopy. Front Hum Neurosci 5, 95.

Funane, T., Atsumori, H., Katura, T., Obata, A. N., Sato, H., Tanikawa, Y., Okada, E., Kiguchi, M., 2014. Quantitative evaluation of deep and shallow tissue layers' contribution to fNIRS signal using multi-distance optodes and independent component analysis. Neuroimage 85 (Part 1), 150-165.

Funane, T., Kiguchi, M., Atsumori, H., Sato, H., Kubota, K., Koizumi, H., 2011. Synchronous activity of two people's prefrontal cortices during a cooperative task measured by simultaneous near-infrared spectroscopy. J Biomed Opt 16 (7), 077011.

Gagnon, L., Cooper, R. J., Yücel, M. A., Perdue, K. L., Greve, D. N., Boas, D. A., 2012. Short separation channel location impacts the performance of short channel regression in nirs. Neuroimage 
59 (3), 2518-2528.

Gagnon, L., Perdue, K., Greve, D. N., Goldenholz, D., Kaskhedikar, G., Boas, D. A., 2011. Improved recovery of the hemodynamic response in diffuse optical imaging using short optode separations and state-space modeling. Neuroimage 56 (3), 1362-1371.

Gagnon, L., Yücel, M. A., Dehaes, M., Cooper, R. J., Perdue, K. L., Selb, J., Huppert, T. J., Hoge, R. D., Boas, D. A., 2012. Quantification of the cortical contribution to the NIRS signal over the motor cortex using concurrent NIRS-fMRI measurements. Neuroimage 59 (4), 3933-3940.

Gilbert, S. J., Spengler, S., Simons, J. S., Steele, J. D., Lawrie, S. M., Frith, C. D., Burgess, P. W., 2006. Functional specialization within rostral prefrontal cortex (area 10): a meta-analysis. J Cogn Neurosci 18 (6), 932-948.

Gohel, S. R., Biswal, B. B., 2015. Functional integration between brain regions at rest occurs in multiple-frequency bands. Brain Connect 5 (1), 23-34.

Grinsted, A., Moore, J. C., Jevrejeva, S., 2004. Application of the cross wavelet transform and wavelet coherence to geophysical time series. Nonlinear Processes in Geophysics $11(5 / 6), 561-566$.

Haeussinger, F. B., Dresler, T., Heinzel, S., Schecklmann, M., Fallgatter, A. J., Ehlis, A.-C., 2014. Reconstructing functional near-infrared spectroscopy (fNIRS) signals impaired by extra-cranial confounds: an easy-to-use filter method. Neuroimage 95, 69-79.

Hari, R., Kujala, M. V., 2009. Brain basis of human social interaction: from concepts to brain imaging. Physiol Rev 89 (2), 453-479. 
Hasson, U., Ghazanfar, A. A., Galantucci, B., Garrod, S., Keysers, C., 2012. Brain-to-brain coupling: a mechanism for creating and sharing a social world. Trends Cogn Sci 16 (2), 114-121.

He, B. J., Zempel, J. M., Snyder, A. Z., Raichle, M. E., 2010. The temporal structures and functional significance of scale-free brain activity. Neuron 66 (3), $353-369$.

Holper, L., Goldin, A. P., Shalóm, D. E., Battro, A. M., Wolf, M., Sigman, M., 2013. The teaching and the learning brain: A cortical hemodynamic marker of teacher-student interactions in the socratic dialog. International Journal of Educational Research 59, 1-10.

Holper, L., Scholkmann, F., Wolf, M., 2012. Between-brain connectivity during imitation measured by fNIRS. Neuroimage 63 (1), 212-222.

Homan, R. W., Herman, J., Purdy, P., 1987. Cerebral location of international 10-20 system electrode placement. Electroencephalography and Clinical Neurophysiology 66 (4), 376-382.

Huppert, T. J., Diamond, S. G., Franceschini, M. A., Boas, D. A., 2009. Homer: a review of time-series analysis methods for near-infrared spectroscopy of the brain. Applied Optics 48 (10), D280-D298.

Hutchison, R. M., Womelsdorf, T., Allen, E. A., Bandettini, P. A., Calhoun, V. D., Corbetta, M., Della Penna, S., Duyn, J. H., Glover, G. H., Gonzalez-Castillo, J., Handwerker, D. A., Keilholz, S., Kiviniemi, V., Leopold, D. A., de Pasquale, F., Sporns, O., Walter, M., Chang, C., 2013. Dynamic functional connectivity: Promise, issues, and interpretations. Neuroimage 80, 360-378.

Izzetoglu, M., Chitrapu, P., Bunce, S., Onaral, B., 2010. Motion artifact cancellation in NIR 
spectroscopy using discrete kalman filtering. Biomed Eng Online 9, 16.

Jiang, J., Chen, C., Dai, B., Shi, G., Ding, G., Liu, L., Lu, C., 2015. Leader emergence through interpersonal neural synchronization. Proc Natl Acad Sci U S A 112 (14), 4274-4279.

Jiang, J., Dai, B., Peng, D., Zhu, C., Liu, L., Lu, C., 2012. Neural synchronization during face-to-face communication. J Neurosci 32 (45), 16064-16069.

Katura, T., Tanaka, N., Obata, A., Sato, H., Maki, A., 2006. Quantitative evaluation of interrelations between spontaneous low-frequency oscillations in cerebral hemodynamics and systemic cardiovascular dynamics. Neuroimage 31 (4), 1592-1600.

Kawasaki, M., Yamada, Y., Ushiku, Y., Miyauchi, E., Yamaguchi, Y., 2013. Inter-brain synchronization during coordination of speech rhythm in human-to-human social interaction. Sci Rep 3, 1692.

Kirilina, E., Jelzow, A., Heine, A., Niessing, M., Wabnitz, H., Brühl, R., Ittermann, B., Jacobs, A. M., Tachtsidis, I., 2012. The physiological origin of task-evoked systemic artefacts in functional near infrared spectroscopy. Neuroimage 61 (1), 70-81.

Kohno, S., Miyai, I., Seiyama, A., Oda, I., Ishikawa, A., Tsuneishi, S., Amita, T., Shimizu, K., 2007. Removal of the skin blood flow artifact in functional near-infrared spectroscopic imaging data through independent component analysis. Journal of Biomedical Optics 12 (6), 062111-1-9.

Koike, T., Tanabe, H. C., Okazaki, S., Nakagawa, E., Sasaki, A. T., Shimada, K., Sugawara, S. K., Takahashi, H. K., Yoshihara, K., Bosch-Bayard, J., Sadato, N., 2016. Neural substrates of shared 
attention as social memory: A hyperscanning functional magnetic resonance imaging study.

Neuroimage 125, 401-412.

Koike, T., Tanabe, H. C., Sadato, N., 2015. Hyperscanning neuroimaging technique to reveal the "two-in-one" system in social interactions. Neurosci Res 90, 25-32.

Konvalinka, I., Bauer, M., Stahlhut, C., Hansen, L. K., Roepstorff, A., Frith, C. D., 2014. Frontal alpha oscillations distinguish leaders from followers: Multivariate decoding of mutually interacting brains. Neuroimage 94, 79-88.

Konvalinka, I., Roepstorff, A., 2012. The two-brain approach: how can mutually interacting brains teach us something about social interaction? Front Hum Neurosci 6, 215.

Lindenberger, U., Li, S.-C., Gruber, W., Müller, V., 2009. Brains swinging in concert: cortical phase synchronization while playing guitar. BMC Neurosci 10, 22.

Liu, X., Duyn, J. H., 2013. Time-varying functional network information extracted from brief instances of spontaneous brain activity. Proc Natl Acad Sci U S A 110 (11), 4392-4397.

Liu, Y., San Liang, X., Weisberg, R. H., 2007. Rectification of the bias in the wavelet power spectrum. J Atmos Oceanic Technol 24 (12), 2093-2102.

Luu, S., Chau, T., 2009. Decoding subjective preference from single-trial near-infrared spectroscopy signals. J Neural Eng 6, 016003-1-8.

Matthews, F., Pearlmutter, B. A., Ward, T. E., Soraghan, C., Markham, C., 2008. Hemodynamics for brain-computer interfaces: optical correlates of control signals. IEEE Signal Processing Magazine 25, 
$87-94$.

Molavi, B., Dumont, G. A., 2012. Wavelet-based motion artifact removal for functional near-infrared spectroscopy. Physiol Meas 33 (2), 259-270.

Nakao, T., Matsumoto, T., Shimizu, D., Morita, M., Yoshimura, S., Northoff, G., Morinobu, S., Okamoto, Y., Yamawaki, S., 2013. Resting state low-frequency fluctuations in prefrontal cortex reflect degrees of harm avoidance and novelty seeking: an exploratory NIRS study. Front Syst Neurosci 7, 115.

Obrig, H., Neufang, M., Wenzel, R., Kohl, M., Steinbrink, J., Einhäupl, K., Villringer, A., 2000. Spontaneous low frequency oscillations of cerebral hemodynamics and metabolism in human adults. Neuroimage 12, 623-639.

Oldfield, R. C., 1971. The assessment and analysis of handedness: the Edinburgh inventory. Neuropsychologia 9 (1), 97-113.

Osaka, N., Minamoto, T., Yaoi, K., Azuma, M., Shimada, Y. M., Osaka, M., 2015. How two brains make one synchronized mind in the inferior frontal cortex: fNIRS-based hyperscanning during cooperative singing. Front Psychol 6, 1811.

Palva, J. M., Zhigalov, A., Hirvonen, J., Korhonen, O., Linkenkaer-Hansen, K., Palva, S., 2013. Neuronal long-range temporal correlations and avalanche dynamics are correlated with behavioral scaling laws. Proc Natl Acad Sci U S A 110 (9), 3585-3590.

R Development Core Team, 2008. R: A Language and Environment for Statistical Computing. R 
Foundation for Statistical Computing, Vienna, Austria, ISBN 3-900051-07-0.

Robertson, F., Douglas, T., Meintjes, E., 2010. Motion artefact removal for functional near infrared spectroscopy: a comparison of methods. IEEE Trans Biomed Eng 57 (6), 1377-1387.

Roggan, A., Friebel, M., Do Rschel, K., Hahn, A., Mu Ller, G., 1999. Optical properties of circulating human blood in the wavelength range 400-2500 nm. J Biomed Opt 4 (1), 36-46.

Saager, R. B., Berger, A. J., 2005. Direct characterization and removal of interfering absorption trends in two-layer turbid media. J Opt Soc Am A Opt Image Sci Vis 22 (9), 1874-1882.

Saager, R. B., Telleri, N. L., Berger, A. J., 2011. Two-detector corrected near infrared spectroscopy (C-NIRS) detects hemodynamic activation responses more robustly than single-detector NIRS. Neuroimage 55 (4), 1679-1685.

Sanger, J., Müller, V., Lindenberger, U., 2012. Intra- and interbrain synchronization and network properties when playing guitar in duets. Front Hum Neurosci 6, 312.

Sasai, S., Homae, F., Watanabe, H., Taga, G., 2011. Frequency-specific functional connectivity in the brain during resting state revealed by NIRS. Neuroimage 56 (1), 252-257.

Schilbach, L., Timmermans, B., Reddy, V., Costall, A., Bente, G., Schlicht, T., Vogeley, K., 2013. Toward a second-person neuroscience. Behav Brain Sci 36 (4), 393-414.

Schippers, M. B., Roebroeck, A., Renken, R., Nanetti, L., Keysers, C., 2010. Mapping the information flow from one brain to another during gestural communication. Proc Natl Acad Sci U S A 107 (20), 9388-9393. 
Scholkmann, F., Holper, L., Wolf, U., Wolf, M., 2013. A new methodical approach in neuroscience: assessing inter-personal brain coupling using functional near-infrared imaging (fNIRI) hyperscanning. Front Hum Neurosci 7, 813.

Scholkmann, F., Kleiser, S., Metz, A. J., Zimmermann, R., Mata Pavia, J., Wolf, U., Wolf, M., 2014. A review on continuous wave functional near-infrared spectroscopy and imaging instrumentation and methodology. Neuroimage 85 Pt 1, 6-27.

Stephens, G. J., Silbert, L. J., Hasson, U., 2010. Speaker-listener neural coupling underlies successful communication. Proc Natl Acad Sci U S A 107, 14425-14430.

Tagliazucchi, E., Balenzuela, P., Fraiman, D., Chialvo, D. R., 2012. Criticality in large-scale brain fMRI dynamics unveiled by a novel point process analysis. Front Physiol 3, 15.

Takahashi, T., Takikawa, Y., Kawagoe, R., Shibuya, S., Iwano, T., Kitazawa, S., 2011. Influence of skin blood flow on near-infrared spectroscopy signals measured on the forehead during a verbal fluency task. Neuroimage 57 (3), 991-1002.

Tanabe, H. C., Kosaka, H., Saito, D. N., Koike, T., Hayashi, M. J., Izuma, K., Komeda, H., Ishitobi, M., Omori, M., Munesue, T., Okazawa, H., Wada, Y., Sadato, N., 2012. Hard to "tune in": neural mechanisms of live face-to-face interaction with high-functioning autistic spectrum disorder. Front Hum Neurosci 6, 268.

Toronov, V., Webb, A., Choi, J. H., Wolf, M., Michalos, A., Gratton, E., Hueber, D., 2001. Investigation of human brain hemodynamics by simultaneous near-infrared spectroscopy and 
functional magnetic resonance imaging. Med Phys 28 (4), 521-527.

Toronov, V., Webb, A., Choi, J. H., Wolf, M., Safonova, L., Wolf, U., Gratton, E., 2001. Study of local cerebral hemodynamics by frequency-domain near-infrared spectroscopy and correlation with simultaneously acquired functional magnetic resonance imaging. Opt Express 9 (8), 417-427.

Torrence, C., Compo, G. P., 1998. A practical guide to wavelet analysis. Bulletin of the American Meteorological society $79(1), 61-78$.

Wei, L., Duan, X., Zheng, C., Wang, S., Gao, Q., Zhang, Z., Lu, G., Chen, H., 2014. Specific frequency bands of amplitude low-frequency oscillation encodes personality. Hum Brain Mapp $35(1), 331-339$.

Xue, S.-W., Li, D., Weng, X.-C., Northoff, G., Li, D.-W., 2014. Different neural manifestations of two slow frequency bands in resting functional magnetic resonance imaging: A systemic survey at regional, interregional, and network levels. Brain Connect 4 (4), 242-255.

Yu, R., Chien, Y.-L., Wang, H.-L. S., Liu, C.-M., Liu, C.-C., Hwang, T.-J., Hsieh, M. H., Hwu, H.-G., Tseng, W.-Y. I., 2014. Frequency-specific alternations in the amplitude of low-frequency fluctuations in schizophrenia. Hum Brain Mapp 35 (2), 627-637.

Zhang, H., Zhang, Y.-J., Lu, C.-M., Ma, S.-Y., Zang, Y.-F., Zhu, C.-Z., 2010. Functional connectivity as revealed by independent component analysis of resting-state fNIRS measurements. Neuroimage 51 (3), 1150-1161.

Zhang, Q., Strangman, G. E., Ganis, G., 2009. Adaptive filtering to reduce global interference in 
non-invasive NIRS measures of brain activation: How well and when does it work? Neuroimage 45 (3), 788-794.

Zhang, Y., Brooks, D. H., Franceschini, M. A., Boas, D. A., 2005. Eigenvector-based spatial filtering for reduction of physiological interference in diffuse optical imaging. Journal of Biomedical Optics $10(1), 11014$.

Zuo, X.-N., Martino, A. D., Kelly, C., Shehzad, Z. E., Gee, D. G., Klein, D. F., Castellanos, F. X., Biswal, B. B., Milham, M. P., 2010. The oscillating brain: complex and reliable. Neuroimage 49 (2), 1432-1445. 